\title{
Rotational tracer diffusion in binary colloidal sphere mixtures
}

\author{
Gijsberta H. Koenderink, ${ }^{1, *}$ Haiyan Zhang, ${ }^{2}$ Minne P. Lettinga, ${ }^{3}$ Gerhard Nägele, ${ }^{3}$ and Albert P. Philipse ${ }^{1}$ \\ ${ }^{1}$ Van't Hoff Laboratory, Debye Institute, Utrecht University, 3584 CH Utrecht, The Netherlands \\ ${ }^{2}$ Department of Physics, Shanghai Jiao Tong University, Shanghai 200030, People's Republic of China \\ ${ }^{3}$ Institut für Festkörperforschung, Forschungszentrum Jülich, D-52425 Jülich, Germany
}

(Received 17 October 2000; published 24 July 2001)

\begin{abstract}
We demonstrate that tracer/host size asymmetry and electrostatic interactions strongly affect rotational self-diffusion in binary mixtures of charged colloidal tracer and host spheres. Tracer diffusion coefficients, measured with time-resolved phosphorescence anisotropy, are compared with calculations of rotational diffusion including two- and three-particle hydrodynamic interactions. We also show that the inverse dependence of the rotational diffusion coefficient on the suspension viscosity is approached only at large size ratios.
\end{abstract}

DOI: 10.1103/PhysRevE.64.022401

PACS number(s): 82.70.Dd, 05.40.Jc

Rotational diffusion of colloidal tracer spheres in dense colloidal host fluids is much less understood than other transport properties such as translational diffusion. Translational diffusion is known to depend strongly on size asymmetry for binary mixtures of tracer and host spheres [1] as well as long-range electrostatic repulsions in case of charged particles [2]. Whether size asymmetry and charge effects, which are abundant in synthetic and naturally occurring complex fluids, also affect rotational tracer diffusion significantly is yet unclear. Therefore we report experiments and theory on rotational diffusion in binary mixtures of charged spheres as a function of size ratio, host volume fraction, and ionic strength. We further investigate whether rotational tracer diffusion is related via a Stokes-Einstein relation to the inverse viscosity of the host suspension.

Experiments on rotational diffusion of spheres, until recently, relied on two techniques that can distinguish different sphere orientations, namely, depolarized dynamic light scattering (DDLS) [3] and nuclear magnetic resonance [4]. These techniques, however, require specific tracer particles that are difficult to synthesize, obstructing a systematic variation of tracer/host size ratio and particle interactions. Recently a novel method called time-resolved phosphorescence anisotropy (TPA) was reported, which requires only a dyelabeled tracer. Such labeling is straightforward [5] and can be applied to a variety of tracer colloids, which can be surfacefunctionalized to control the particle interactions [6]. In this way TPA can be applied to tracer rotation in colloidal fluids, porous media [5], or biologically relevant matrices. In this paper we will first outline the TPA technique, then calculate short-time rotational diffusion coefficients and finally compare our experimental and theoretical results.

For the TPA measurements we employ silica tracer spheres (of hydrodynamic radius $a_{T}=96 \mathrm{~nm}$ with polydispersity $\sigma$ of $9 \%$ ) labeled with an eosin dye [5] and charged silica host spheres (of hydrodynamic radii $a_{H}=92 \mathrm{~nm}$ and $\sigma$ $=8 \%$, or $298 \mathrm{~nm}$ and $\sigma=2 \%$ ). The solvent is an optically matching 60-40 v/v mixture of dimethylsulfoxide and $N, N$ dimethylformamide, with a dielectric constant of 43 , and $\mathrm{LiCl}$ added to adjust the ionic strength. The short-time rota-

\footnotetext{
*Corresponding author.
}

tional diffusion coefficient $D_{s}^{r}$ of the tracer was determined by TPA, from the decay of the polarization anisotropy induced by excitation of the dye inside the tracer with a vertically polarized light pulse [5]. The anisotropy relaxation is due to reorientation of the tracer sphere during the lifetime (several $\mathrm{ms}$ ) of the excited state. The experimental time window is ca. 0.01-10 ms. TPA experiments were performed at $T=23{ }^{\circ} \mathrm{C}$ using the experimental setup described in [5]. Anisotropy decay curves were always single exponential as expected for noninteracting, monodisperse tracer spheres [5]. Diffusion data were normalized by the Stokes-Einstein diffusion coefficient $D_{0}^{r}=k_{B} T / 8 \pi \eta_{0} a_{T}^{3}$ of an isolated tracer sphere in a solvent with shear viscosity $\eta_{0}$.

For comparison with experimental results we first calculate the normalized short-time rotational diffusion coefficient $H_{s}^{r}=D_{s}^{r} / D_{0}^{r}$ of a single tracer in a host sphere dispersion as a function of host volume fraction $\phi$ and size ratio $\lambda$ $=a_{T} / a_{H}$. To this end we extend a method for calculating $H_{s}^{r}$ for monodisperse sphere suspensions $[7,8]$ to binary mixtures. For moderate volume fractions, $H_{s}^{r}$ can be written as a sum over hydrodynamic interactions between clusters of two, three, and more particles,

$$
H_{s}^{r}(\phi, \lambda)=1+H_{s 1}^{r}(\phi, \lambda) \phi+H_{s 2}^{r}(\phi, \lambda) \phi^{2}+\cdots .
$$

We truncate this rooted cluster approximation after the third term, so we evaluate only the coefficient $H_{s 1}^{r}(\phi, \lambda)$, representing hydrodynamic interactions between the tracer and one host sphere, and $H_{s 2}^{r}(\phi, \lambda)$, corresponding to a tracer interacting with two host spheres. The coefficient $H_{s 1}^{r}(\phi, \lambda)$ is expressible by the integral

$$
\begin{aligned}
H_{s 1}^{r}(\phi, \lambda)= & \frac{1}{a_{H}^{3}} \int_{a_{H}+a_{T}}^{\infty} d r r^{2} g_{\mathrm{TH}}^{(2)}(r ; \phi, \lambda) \\
& \times\left[\alpha_{\mathrm{TH}}^{r r}(r ; \lambda)+2 \beta_{\mathrm{TH}}^{r r}(r ; \lambda)\right],
\end{aligned}
$$

involving the tracer-host $(\mathrm{TH})$ radial distribution function $g_{\mathrm{TH}}^{(2)}(r ; \phi, \lambda)$ and the two-body rotational hydrodynamic mobility functions $\alpha_{\mathrm{TH}}^{r r}(r ; \lambda)$ and $\beta_{\mathrm{TH}}^{r r}(r ; \lambda)$, which depend on the tracer-host distance $r$ and on $\lambda$. For $\lambda=1$, multipole expansions of these mobility functions in powers of $r^{-1}$ are 
known, in principle, to arbitrary order $[7,8]$. For $\lambda \neq 1$, an explicit $r^{-1}$ expansion up to order $r^{-12}$ has been provided in $[9,10]$. For $H_{s 2}^{r}(\phi, \lambda)$ with $\lambda \neq 1$ we have used the method of reflections and connectors to derive a new result for the asymptotic far-field term in the inverse distance expansion of the three-body rotational mobility tensor (details will be described in [11]). Using this asymptotic form, $H_{s 2}^{r}(\phi, \lambda)$ is given, similar to Eq. (2), by

$$
\begin{aligned}
H_{s 2}^{r}(\phi, \lambda)= & \frac{225}{16}\left(\frac{\lambda}{1+\lambda}\right)^{3} \int_{0}^{1} d t_{12} \int_{0}^{1} d t_{13} \int_{-1}^{1} d \xi_{1} \\
& \times g_{\mathrm{THH}}^{(3)}\left(t_{12}, t_{13}, \xi_{1}\right) \frac{\left(t_{12} t_{13}\right)^{2}}{h^{3 / 2}} f\left(t_{12}, t_{13}, \xi_{1}\right) \\
f\left(t_{12}, t_{13}, \xi_{1}\right)= & \frac{t_{12} t_{13} \xi_{1}\left(1-\xi_{1}^{2}\right)}{h}+\xi_{1}^{2}-1-\frac{2}{h^{2}}\left(t_{12}-t_{13} \xi_{1}\right) \\
& \times\left(t_{13}-t_{12} \xi_{1}\right) \\
& \times\left[\left(t_{12}^{2}+t_{13}^{2}\right) \xi_{1}-t_{12} t_{13}\left(5-3 \xi_{1}\right)\right]
\end{aligned}
$$

with the static triplet distribution function $g_{\mathrm{THH}}^{(3)}$ invoked. This function describes static correlations between a tracer and two host spheres that depend on the reduced distances $t_{1 i}=\left(a_{T}+a_{H}\right) / r_{1 i}$ and the angular cosine $\xi_{1}$ $=\vec{r}_{12} \cdot \vec{r}_{13} /\left(r_{12} r_{13}\right)$, where $\vec{r}_{l 1}=r_{1}-r_{i}$ is the vector pointing from host particle $i$ to the tracer 1. Equation (3) contains hydrodynamic interactions between three rather than two spheres. Note that $h=t_{12}^{2}+t_{13}^{2}-2 \xi_{1} t_{12} t_{13}$. For $\lambda=1$, Eq. (3) reduces to an asymptotic result for $H_{s 2}^{r}(\phi, 1)$ derived recently in [8].

To evaluate Eq. (1) we now need to specify the pair potential between the particles, which determines the functions $g_{\mathrm{TH}}^{(2)}$ and $g_{\mathrm{THH}}^{(3)}$ in Eqs. (2) and (3). For uncharged tracer and host spheres, a virial expansion of $H_{s}^{r}(\phi, \lambda)$ up to quadratic order in $\phi$, viz.

$$
H_{s}^{r}(\phi, \lambda)=1+h_{1}^{r}(\lambda) \phi+h_{2}^{r}(\lambda) \phi^{2}
$$

is obtained by substituting for $g_{\mathrm{THH}}^{(3)}$ in Eq. (3) its zerodensity form $g_{\mathrm{THH}}^{(3)}=\Theta\left(r_{12}-\left[a_{T}+a_{H}\right]\right) \Theta\left(r_{13}-\left[a_{T}\right.\right.$ $\left.\left.+a_{H}\right]\right) \Theta\left(r_{23}-2 a_{H}\right)$, with $\Theta$ denoting the unit step function. For $g_{\mathrm{TH}}^{(2)}$ in Eq. (4) we use the first-order density form as given in [11]. Notice that the second virial coefficient, $h_{2}^{r}(\lambda)$ is the sum of a two-body part due to the $O(\phi)$ contribution of $g_{\mathrm{TH}}^{(2)}$ [cf. Eq. (2)], and a three-body part originating from the zero-density form of $g_{\mathrm{THH}}^{(3)}[\mathrm{cf}$. Eq. (3)].

Figure 1 shows our approximate theoretical results for the first and second virial coefficients $h_{1}^{r}(\lambda)$ and $h_{2}^{r}(\lambda)$ in Eq. (4) for uncharged tracer/host dispersions. The first virial coefficient decreases monotonically with $\lambda$, from $h_{1}^{r}(\lambda=0)$ $=0$ towards $h_{1}^{r}(\lambda \rightarrow \infty)=-2.5$. As shown in the inset of Fig. 1 , the second virial coefficient $h_{2}^{r}(\lambda)$ is nonmonotonic with a minimum at $\lambda \approx 1.2$. Regarding $h_{2}^{r}(\lambda)$, it should be noted that hydrodynamic four-body and higher-order contributions are important at large values of $\lambda$, though they are not accounted for in our theory. For $\lambda=1$ our calculations should

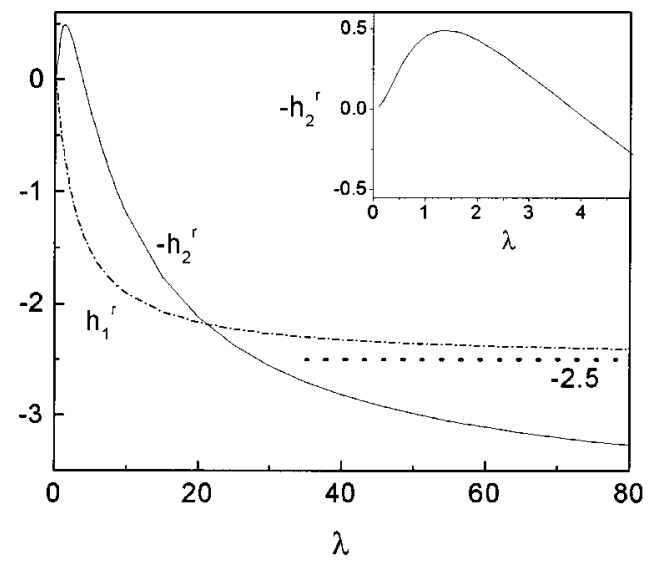

FIG. 1. Calculated first and second virial coefficients $h_{1}^{r}$ and $-h_{2}^{r}$ versus size ratio $\lambda$ for neutral tracer and host spheres. The $\lambda$ dependence of $h_{1}^{r}$ is well parametrized by $h_{1}^{r}(\lambda)=-2.5 /(1$ $\left.+3.0 \lambda^{-1}\right)$.

be compared to previous theoretical work on short-time rotational diffusion in monodisperse sphere suspensions. The most accurate prediction for hard spheres, including hydrodynamic lubrication interactions, due to Cichocki, EkielJezewska, and Wajnryb [8], reads

$$
D_{s}^{r} / D_{0}^{r}=1-0.631 \varphi-0.726 \varphi^{2} \text {. }
$$

We obtain somewhat different values of $h_{1}^{r}=-0.525$ and $h_{2}^{r}=-0.451$ since we account only for $O\left(r^{-12}\right)$ terms in the two-body and for the leading $O\left(r^{-9}\right)$ term in the three-body mobility functions.

From our theoretical results for $h_{1}^{r}$ and $h_{2}^{r}$ (cf. Fig. 1) we expect that rotational diffusion slows down with increasing $\lambda$. This predicted trend is indeed observed in our TPA data for charged tracer spheres dispersed in charged host sphere suspensions, with sufficient salt added to screen electrostatic repulsions (cf. Fig. 2). Figure 2 also includes $H_{s}^{r}$ data for $\lambda$ $=10$ that we have obtained earlier using DDLS on aqueous binary hard-sphere-like suspensions [12]. Clearly, rotational

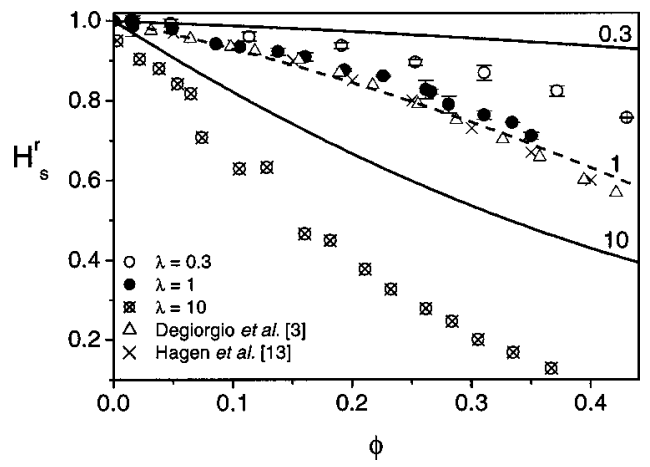

FIG. 2. Normalized rotational tracer diffusion coefficient $H_{s}^{r}$ versus host volume fraction $\phi$ for neutral tracer/host mixtures of size ratios $\lambda=0.33$ (TPA), $\lambda=1$ (TPA), and $\lambda=10$ (DDLS from [12]). Solid lines represent our short-time theoretical predictions to order $\phi^{2}$. For $\lambda=1$ we include $\Delta$, DDLS data on near hard spheres [3]; $\times$, hard sphere simulation results [13], and Eq. (5) [8] (dashed line). 


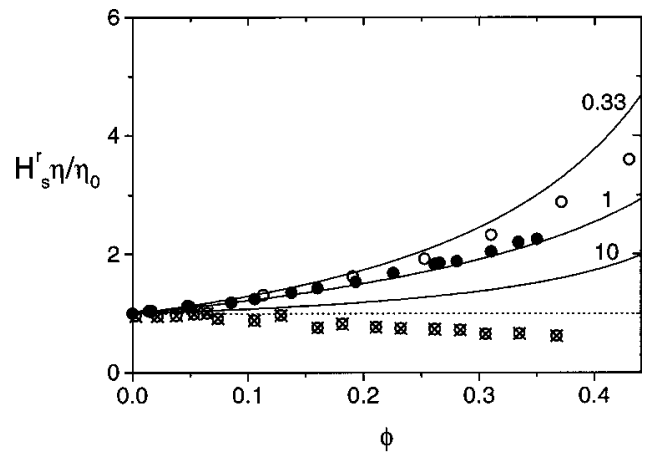

FIG. 3. Product of experimental values for $H_{s}^{r}$ with a semiempirical result for $\eta_{\infty} / \eta_{0}$ [16] at $\lambda=0.33$ and 1 for neutral tracer/host mixtures. For $\lambda=10$ we use a rescaled mode-coupling result for $\eta / \eta_{0}$ [17]. Solid lines represent the theoretical predictions for $H_{s}^{r} \eta_{\infty} / \eta_{0}$. The dotted line represents the GSE, $H_{s}^{r} \eta_{\infty} / \eta_{0}=1$.

tracer diffusion is significantly slowed down with increasing tracer size. For given $\phi_{H}$ and $a_{H}$ there is a larger probability for the tracer to be close to a host particle of its nextneighbor shell when the tracer/host size ratio is increased, leading to a stronger hydrodynamic hindrance of its rotational motion. The data for $\lambda=1$ are in fair agreement with previous experimental results for aqueous near-hard-sphere suspensions [3] and recent simulations [13]. Moreover, our experimental data for $\lambda=1$ agree well with the theoretical expression in Eq. (5) up to $\phi \leqslant 0.35$. The small discrepancy is probably due to residual electrostatic repulsions. For the strongly asymmetric cases $\lambda=0.33$ and $\lambda=10$ the experimental data at larger $\phi$ are significantly below our theoretical predictions. This is partially due to a narrowing of the $\phi$ range where our approximate theoretical treatment applies with increasing size asymmetry: first, for large $\lambda$ a tracer is very likely to interact with two or more host particles at a time, unless $\phi$ is very small. This means that the truncated rooted-cluster expansion in Eq. (1) becomes less accurate with increasing $\lambda$. Second, the truncated inverse distance expansion of the two- and three-body hydrodynamic mobility functions becomes less accurate as $\lambda$ deviates more from 1 . For the largest size ratio $\lambda=10$ one should also note that the experimentally accessible time scale depends on $\lambda$. At large $\lambda$ the tracer diffuses much slower than the host particles (as roughly estimated from $D_{0}^{r} \propto a^{-3}$ ). Consequently, for $\lambda$ $=10$, DDLS actually measures the long-time rather than the short-time rotational tracer diffusion coefficient. Due to memory effects, the long-time coefficient $D_{l}^{r}$ is smaller than the short-time coefficient [14]. No theory is yet available for long-time rotational diffusion in mixtures, so that we can only compare our long-time DDLS data to short-time calculations. It has been shown for $\lambda=1$ and neutral spheres, that the difference between $D_{l}^{r}$ and $D_{s}^{r}$ is small to first order in $\phi$ [15]. However, Fig. 2 suggests that for larger $\lambda$ and larger $\phi$ the difference may become significant.

The $\lambda$ dependence of $h_{1}^{r}$ in Fig. 1, with $h_{1}^{r}(\lambda \rightarrow \infty)=$ -2.5 , suggests that as $\lambda$ becomes very large, a generalized Stokes-Einstein (GSE) relation $H_{s}^{r}=\eta_{0} / \eta$ is approached. Thus, to leading order in $\phi$ and very large $\lambda$, the tracer experiences an effective fluid viscosity given by the Einstein
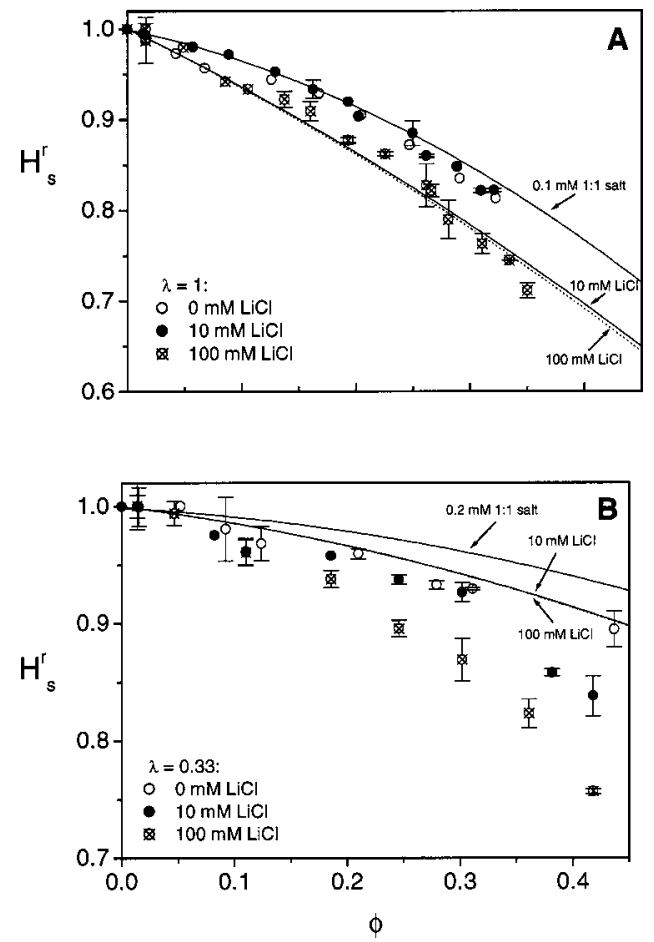

FIG. 4. TPA (circles) and TRC-RY (lines) results for $H_{s}^{r}$ versus host volume fraction $\phi$ for (a) $\lambda=1$ using $Z_{T}=Z_{H}=220, a_{T}$ $=90 \mathrm{~nm}$, and $0.1 \mathrm{mM}$ residual $1-1$ electrolyte, and (b) $\lambda=0.33$, using $Z_{T}=220, Z_{H}=1200$, and $0.2 \mathrm{mM}$ residual electrolyte. The TRC-RY parameters are chosen compatible with the observed freezing volume fractions. It should be noted that no colloidal crystallization was observed during the experiments.

equation $\eta / \eta_{0}=1+2.5 \phi$. To check whether corresponding large- $\lambda$ GSE relations are valid also at larger values of $\phi$, we multiply the experimental $H_{s}^{r}$ data in Fig. 2 by the relative viscosity of the host suspension and replot them in Fig. 3. For $\lambda=0.33$ and 1 , the short-time $H_{s}^{r}(\phi, \lambda)$ data are multiplied by the corresponding normalized high-frequency viscosity $\eta_{\infty}(\phi) / \eta_{0}$, as given by an accurate semiempirical expression in [16]. The long-time experimental data for $H_{1}^{r}(\phi, \lambda)$ at $\lambda=10$ are multiplied instead by the relative zero-shear ("long-time") viscosity $\eta(\phi) / \eta_{0}$ calculated using a rescaled mode-coupling scheme that agrees well with experimental data for hard spheres [17]. As $\lambda$ increases, both the theoretical and the experimental rotational diffusion data are clearly seen to approach the GSE.

From suspensions of uncharged particles we now move to charge-stabilized tracer/host mixtures at various ionic strengths. In case of long-range electrostatic repulsions, only the leading far-field terms of the mobility functions are needed in the expressions for $H_{s 1}^{r}(\phi, \lambda)$ and $H_{s 2}^{r}(\phi, \lambda)$, since $g_{\mathrm{TH}}^{(2)}(r)$ is practically zero at small $r$ [18]. However, contrary to hard spheres, it is now necessary to determine even at small $\phi$ radial distribution functions from the accurate Rogers-Young (RY) integral equation scheme for the binary macrofluid model (cf. [14]). In this model the pair potential between two charged colloidal spheres consists of a hard sphere supplemented by a screened DLVO (DerjaguinLandau-Verwey-Overbeek) potential $[14,18]$. For the static 
triplet function in $H_{s 2}^{r}(\phi, \lambda)$ we use Kirkwood's superposition approximation $g_{\mathrm{THH}}^{(3)}=g_{\mathrm{TH}}^{(2)}\left(r_{12}\right) g_{\mathrm{TH}}^{(2)}\left(r_{13}\right) g_{\mathrm{HH}}^{(2)}\left(r_{23}\right)$. In our RY calculations of $g_{\mathrm{TH}}^{(2)}$ and $g_{\mathrm{HH}}^{(2)}$, we assume that the effective charges $Z_{H}$ and $Z_{T}$ of the host and tracer particles are independent of host volume fraction and ionic strength. Our approximate calculation of $H_{s}^{r}$ for charge-stabilized systems will be denoted as the TRC-RY (i.e. truncated rooted cluster$\mathrm{RY)}$ method.

In Fig. 4(a) we compare experimental and TRC-RY results for $H_{s}^{r}$ of charge-stabilized particles at $\lambda=1$ for various $\mathrm{LiCl}$ concentrations. Experiments and theory are in qualitative agreement, showing that for fixed $\phi, H_{s}^{r}$ decreases with increasing ionic strength. This ionic strength dependence manifests that increasing electrostatic screening strengthens hydrodynamic particle coupling since the particle separation is reduced. Interestingly, the experimental ionic strength dependence is somewhat different from that predicted by our calculations. The TPA data show that rotational diffusion for 0 and $10 \mathrm{mM}$ added salt is similar, while adding $100 \mathrm{mM}$ $\mathrm{LiCl}$ leads to a significant reduction of $H_{s}^{r}$. Theory, however, indicates that $H_{s}^{r}$ at 10 and $100 \mathrm{mM}$ added $\mathrm{LiCl}$ is comparable, since both systems are hard-sphere-like due to the small Debye screening lengths of $\kappa^{-1}=0.026 a_{H}$ and $\kappa^{-1}$ $=0.008 a_{H}$, respectively. The discrepancy may stem from details in the experimental interaction potential (e.g., solvation effects), which are not addressed in our calculations. Figure 4(b) illustrates the effect of lowering $\lambda$ from 1 to 0.33 . While the ionic strength dependence of $H_{s}^{r}$ follows the same trends as for $\lambda=1$, the hindrance of rotational diffusion at a given ionic strength is now considerably smaller, as observed also for hard spheres (cf. Fig. 2). The TRC-RY results for $H_{s}^{r}(\lambda=0.33)$ at larger $\phi$ are consistently larger than the experimental data. This observation can be partially attributed to the fact that the $\phi$ range where our approximate theoretical treatment of $H_{s}^{r}$ applies substantially narrows as $\lambda$ deviates more from 1. Moreover, we made simplifying assumptions concerning the density and ionic strength dependence of the effective charges $Z_{T}$ and $Z_{H}$. Notice also that the experimental $\lambda$ contains polydispersity effects. For example, a slightly larger value $\lambda=0.4$ used in the TRC-RY method significantly improves agreement with the experimental data.

To conclude, we have quantitatively investigated the effects of size asymmetry, host concentration, and ionic strength on rotational tracer diffusion. Both the experimentally observed strong dependence of $H_{s}^{r}$ on size ratio and ionic strength, and the approach towards a GSE behavior with increasing size ratio, agree (at least qualitatively) with our approximate theory for rotational diffusion. Future work will focus on the yet unresolved distinction between shortand long-time rotational self-diffusion, as well as the possibly large influence of interaction potential details on rotational diffusion.

We thank S. Sacanna, M. Pazzini, and C. van Kats for particle preparation, B. D'Aguanno and J. Bergenholtz for providing the RY code, and J. Bergenholtz, M. Watzlawek, and $\mathrm{H}$. Ma for helpful discussions. This work was supported by CW/NWO (The Netherlands), the Deutsche Forschungsgemeinschaft (SFB 513), and the National Natural Science Foundation of China.
[1] A. Imhof and J. K. G. Dhont, Phys. Rev. E 52, 6344 (1995).

[2] M. Watzlawck and G. Nägele, Phys. Rev. E 56, 1258 (1997).

[3] V. R. Degiorgio, R. Piazza, and R. B. Jones, Phys. Rev. E 52, 2707 (1995).

[4] A. Tolle and H. Sillescu, Langmuir 10, 4420 (1994).

[5] M. P. Lettinga, C. M. van Kats, and A. P. Philipse, Langmuir 16, 6166 (2000).

[6] A. P. Philipse and A. Vrij, J. Colloid Interface Sci. 128, 121 (1989).

[7] R. B. Jones, Physica A 150, 339 (1988).

[8] B. Cichocki, M. L. Ekiel-Jezewska, and E. Wajnryb, J. Chem. Phys. 111, 3265 (1999).

[9] D. J. Jeffrey and Y. Onishi, J. Fluid Mech. 139, 261 (1984).
[10] P. Mazur and W. van Saarloos, Physica A 115, 21 (1982).

[11] H. Zhang and G. Nägele (unpublished).

[12] G. H. Koenderink and A. P. Philipse, Langmuir 16, 5631 (2000).

[13] M. H. J. Hagen, D. Frenkel, and C. P. Lowe, Physica A 272, 376 (1999).

[14] G. Nägele, Phys. Rep. 272, 215 (1996).

[15] R. B. Jones, Physica A 157, 752 (1989).

[16] R. A. Lionberger and W. B. Russel, J. Rheol. 38, 1885 (1994).

[17] A. J. Banchio, J. Bergenholtz, and G. Nägele, Phys. Rev. Lett. 82, 1792 (1999).

[18] M. Watzlawek and G. Nägele, Physica A 235, 56 (1997). 